\title{
Importance volumétrique des œufs chez les Rotifères planctoniques
}

\author{
R. Pourriot 1 \\ C. Rougier'
}

Mots clés : Rotifères, plancton, volume des œufs, biovolume.

Les rapports volumétriques entre l'œuf amictique $\left(V_{0}\right)$ et la femelle adulte $\left(V_{A}\right)$ sont établis et discutés chez 21 populations naturelles ou expérimentales appartenant à 15 espèces.

Chez les Rotifères herbivores, la relation allométrique est décrite par l'équation : $V_{0}=3,62 V_{A} 0,816$. L'exposant de celle-ci est très proche de la valeur générale 0,75 reliant l'investissement reproductif à la taille. Une relation intraspécifique similaire est observée chez $B$. calyciflorus. Le ratio $\mathrm{V}_{0} / \mathrm{V}_{\mathrm{A}}$ est moins variable chez les Asplanchna ovovivipares dont le rapport du poids sec au poids frais $(4 \%)$ est notablement inférieur à celui des autres especces $(>=10 \%)$.

En outre, des relations allométriques similaires ont été calculées entre le volume des œufs de durée et celui des ceufs mâles d'une part, et le volume de la mère d'autre part, chez, respectivement, sept et trois des espèces précédentes. Les écarts observés sont interprétés comme le résultat de contraintes sélectives différentes.

\section{Volumetric importance of the eggs in planktonic rotifers}

Keywords : Rotifera, plankton, eggs volume, biovolume.

The relationships between amictic egg volume $\left(\mathrm{V}_{0}\right)$ and adult female volume $\left(\mathrm{V}_{\mathrm{A}}\right)$ have been established and discussed in 21 natural of laboratory populations of planktonic rotifers belonging to 15 species.

In the herbivorous rotifers, the allometric relationship is described by the following equation : $V_{0}=3.62 V_{o} 0.816$. The exponent of the equation is close to the general value of 0.75 . Similarly to the interspecific relationships, the egg to volume ratios vary within a rotifer species (ex. Brachionus calyciflorus). These relationships differ in the ovoviviparous genus Asplanchna, with a relative dry mass content ( $4 \%$ of the fresh weight) notably lower than that observed in other species.

Similar allometric relations have also been found between the volume of resting eggs or of male eggs and the volume of their mothers in seven and three of the previous species respectively. Some differences are interpreted as resulting from various selective constraints.

\section{Introduction}

Quiconque a observé des femelles ovigères de Rotifères a pu constater l'énorme volume des œufs femelles par rapport à celui de la femelle (fig. 1). $L$ a rapidité de la croissance de ces organismes dont la maturité sexuelle est atteinte après une courte phase de croissance juvénile (environ un jour à 20 ${ }^{\circ} \mathrm{C}$, ex. in Pourriot 1973) est liée à cette faible différence de taille entre l'œuf (donc le nouveau-né) et l'adulte.

Une relation allométrique relie le volume de l'œuf $\left(V_{0}\right)$ et le volume du corps de l'adulte $\left(V_{A}\right)$ suivant

1. Université Paris 6, Laboratoire de Géologie Appliquée, B 123, F-75252 Paris Cédex. la formule générale $\mathrm{V}_{\mathrm{o}}=\mathrm{a} \mathrm{V}_{\mathrm{A}}^{\mathrm{b}}$ (cf. Pauli 1989); le rapport $V_{o} / V_{A}$ est d'autant plus élevé que l'espèce est de plus petite taille. Selon Pauli (1989), chez 12 especes du Lac de Constance, cette relation se traduit par l'équation :

$$
\mathrm{V}_{\mathrm{O}}=1272 . \mathrm{V}_{\mathrm{A}} 0,3379 \text {. }
$$

Comme le note l'auteur, la valeur de l'exposant est étonnamment faible par comparaison avec d'autres invertébrés où l'investissement reproductif absolu croît avec la puissance 0,75 de la taille (Peters 1983).

Le présent travail a pour objectif d'établir et de discuter ces relations pour 21 populations correspondant à 15 espèces de Rotifères planctoniques en provenance de quelques milieux de la région parisienne et plus particulièrement du lac de Créteil. 


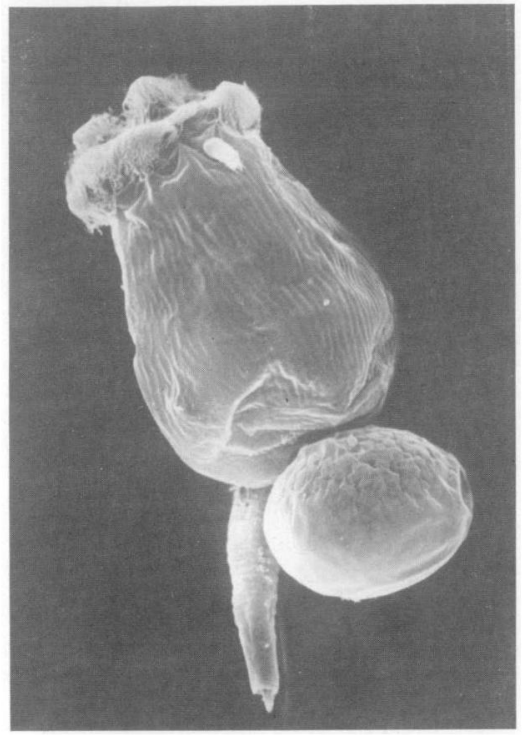

Fig-1. Femelle amictique de Brachionus (B. sericus) portant un ouf, photographiée au miçoscope èlectronique à balayage (document P. Clément).

Fig. 1. Brachionus (B. sericus) amictic female carrying an egg (SEM photograph by P. Clément).

Six des clones élevés au laboratoire et une population naturelle ayant produit des oufs de durée, nous avons examiné également les relations existant entre leur volume et celui de la femelle mère. En complément, nous avons aussi comparé le volume des œufs mâles et celui de la femelle adulte chez les seuls clones algivores (la mesure de la taille des oufs mâles s'avèrant délicate chez les $A$ splanchna carnivores ovovivipares).

\section{Matériel et méthodes}

Des vingt et une populations étudiées, neuf proviennent du plancton estival du lac de Créteil (1984-1986) ; une, du plancton d'une retenue de stockage (Méry/Oise) et toutes les autres, de cultures d'origines variées (Tableau I).
Les conditions thermique: au moment de la ponte avoisinent $20-22{ }^{\circ} \mathrm{C}$, ce qui réduit considérablement l'influence propre au facteur température.

Les volumes des œufs et des adultes ont été cal culés en assimilant ceux-ci à des figures géométri. ques simples, le plus souvent à un ellipsoïde de révolution, plus rarement à un cône (Hexarthra) ou à un parallélépipède (Keratella quadrata, Polyarthra) selon la méthode préconisée par Ruttner-Kolisko (1977). Nous avons utilisé pour nos calculs soit la formule simplifiée de cet auteur, soit une formule recalculée d'après les mensurations effectuées sur nos échantillons (Tableau II).

Les mesures ont été faites au microscope sur des lots de 20 à 30 individus provenant d'échantillons fixés au formol à $5 \%$, sauf pour quatre espèces au tégument trop déformable, Epiphanes brachionus, Asplanchna girodi, A. brightwelli et A. herricki, mesurées sur des individus vivants, juste immobilisés.

Seule la population naturelle d'Asplanchna priodonta récoltée dans le lac de Créteil a fourni des ceufs de durée en quantité suffisante pour leur étude. Les clones de laboratoire ayant produit des ceufs mâles et des oufs de durée sont les mêmes que ceux précédemment étudiés et les conditions identiques (Tableau III).

Les mêmes formules ont été utilisées pour le calcul des volumes des femelles adultes, amictiques ou mictiques, et de leurs oufs.

\section{Résultats}

1. Relations allométriques entre le volume de l'ceuf amictique et le volume de l'adulte

Pour les 21 populations étudiées, les volumes moyens des œufs, $V_{0}$, sont significativement corrélés à ceux des femelles adultes, $V_{A},\left(r^{2}=0,598, p\right.$ $<0,01$ ) et la valeur de l'exposant $b$ de l'équation générale est de 0,386. Cette faible valeur est proche de celle obtenue par Pauli (1989). Toutefois, l'examen de la représentation graphique de cette régression montre une nette rupture de pente au delà d'un volume de la femelle $>4.000 .000{ }_{\mu} \mathrm{m}^{3}(\mathrm{Ln}>15,2)$, les valeurs les plus élevées se différenciant nettement des autres en se situant plutôt autour d'un plateau (fig, 2). Celles-ci correspondant aux Asplanchna, espèces prédatrices qui présentent d'autres caractères très particuliers (voir discussion). 
Tableau I. Populations ou clones utilisés, origine, taille de la femelle adulte ( $L_{A}$ ) et grand diamétre de l'auuf femelle parthénogénétique (Lo) ; * données de Pourrjot (1973).

Table I. Populations or clones, origin, mean size of the adult female (LA) and mean great diameter of the amitic egs.

\begin{tabular}{|c|c|c|c|c|}
\hline espèce & origine & $t{ }^{\circ} \mathrm{C}$ & $\mathrm{LA}_{\mathrm{A}} \pm$ e.s. HIr & Los \pm e.s. $\mu$ m \\
\hline \multicolumn{5}{|l|}{ Keratella cochlearis } \\
\hline cochlearis & lac de Creteil & 22 & $82.6 \pm 1.9$ & $54,9 \pm 1.8$ \\
\hline$\underline{K} \cdot \underline{c}$ I Iobusta & id. & 22 & $110,8 \pm 2,4$ & $67,4 \pm 2,2$ \\
\hline K. $\underline{\text { c. }}$ tecta & bassin de Méry & & $94,1 \pm 3,0$ & $57,1 \pm 2,8$ \\
\hline K. quadrata & lac de Créteil & 20 & $145.5 \pm 3,9$ & $87,9 \pm 4,3$ \\
\hline Polyarthra vulgaris & id. & 22 & $110.2 \pm 4.3$ & $72.1 \pm 2.9$ \\
\hline Filinia lonqiseta & id. & 22 & $146,1 \pm 6,4$ & $72,4 \pm 6.8$ \\
\hline Hexarthra mira & id. & 22 & $190,0 \pm 8,7$ & $83,8 \pm 5,2$ \\
\hline Brachionus anquiaris & id. & 22 & $108.7 \pm 3.3$ & $62,1 \pm 5,7$ \\
\hline B. anqularis CR89 & culture & 24 & $127,8 \pm 3,9$ & $84,4 \pm 3,4$ \\
\hline B. calyciflorus & Iac de Créteil & 19 & $247.8 \pm 7.5$ & $138,8 \pm 7,2$ \\
\hline B. calyciflorus A89 & culture & 20 & $208,7 \pm 8,0$ & $134,0 \pm 7,4$ \\
\hline B. calrciflorus $B L$ & id $\cdot *$ & 20 & $188,6 \pm 12,6$ & $124,1 \pm 7,1$ \\
\hline B. calyciflorus LC & $i d \cdot \star$ & 20 & $222.7 \pm 10,8$ & $127,3 \pm 5,3$ \\
\hline B. calyciflorus $M B$ & $i d \cdot \star$ & 20 & $229.6 \pm 9.7$ & $134,9 \pm 6,7$ \\
\hline B. plicatilis $\mathrm{COB}$ & culture & 20 & $168,1 \pm 8,0$ & $96,6 \pm 5,1$ \\
\hline B. plicatilis GS74 & id. & 20 & $251,3 \pm 8,3$ & $130,9 \pm 9,6$ \\
\hline Eplphanes brachionus & $1 d$. & 20 & $293,0 \pm 11,8$ & $124,6 \pm 6,1$ \\
\hline Asplanchna prigdonta & lac de Créteil & 20 & $404,4 \pm 23,9$ & $86,6 \pm 7,7$ \\
\hline A. girodi & culture & 20 & $779,0 \pm 87,2$ & $127,8 \pm 8,0$ \\
\hline A. brightwel11 & id. & 20 & $838,0 \pm 87,5$ & $121,3 \pm 10,9$ \\
\hline A. herricki & id. & 20 & $966,3 \pm 59,7$ & $118,8 \pm 9,6$ \\
\hline
\end{tabular}


Tableau II. Formules de calcul du volume de la femelle adulte $\left(V_{\mathrm{A}}\right)$, volume de l'cuf amictique $\left(V_{0}\right)$ et ratio $V_{0} / V_{A}$ calculé d'après la formule simplifiée ${ }^{*}$ ) ou la formule recalculée $\left(\mathrm{L}_{1}=\right.$ longueur totale, épine postérieure comprise ; $\mathrm{L}=$ longueur du corps, sans épine). Les volumes indiqués correspondent (sauf pour $B$. calyciflorus BL, LC, MB) à la moyenne des volumes individuels et non aux volumes d'un individu (ou d'un ceuf) de taille moyenne.

Table II. Formulas for calculating the volume of adult fernales, mean values of this volume (VA), mean volume of the amictic ey and ratio $\mathrm{V}_{\mathrm{o}} / \mathrm{V}_{\mathrm{A}}\left\langle\mathrm{L}_{\mathrm{t}}=\right.$ total lenght, posterior spine included $\mathrm{L}=$ body lenght without posterior spines).

\begin{tabular}{|c|c|c|c|c|c|c|}
\hline espéce & $\begin{array}{l}\text { fornule simpiifice } \\
\text { de RUTWNER-ROLISXO }\end{array}$ & $\begin{array}{c}V_{0} / V_{n} \\
*\end{array}$ & formle utilisee & $V_{2} \mu \pi^{3}$ & $V_{0} n^{3}$ & $V_{0} / V_{n}$ \\
\hline$\frac{\text { Keratella }}{\text { cochlearis }}$ & $V=0,02 \mathrm{Lt}^{3}$ & 3,79 & $V=0,17 \mathrm{~L}^{3}$ & 96021 & 14394 & 0,46 \\
\hline$\underline{R} \cdot \underline{\underline{c}}$ Iobustå & $V=0,02 \mathrm{lt}^{3}$ & 0,74 & $V=0,17 \mathrm{i}^{3}$ & 231577 & 100621 & 0,44 \\
\hline$\underline{\underline{R}} . \underline{\text { c. tectad }}$ & & & $v=0,17 \mathrm{i}^{3}$ & 142020 & 53154 & 0,38 \\
\hline K. quadrata & $V=0,22 \mathrm{~b}^{3}$ & 0,30 & $V=0.22 \mathrm{~b}^{3}$ & 678812 & 204516 & 0,30 \\
\hline Polyarthra vulaaris & $V=0,28 \mathrm{~L}^{3}$ & 0,22 & $V=0,28 \mathrm{~L}^{3}$ & 376183 & 81422 & 0,22 \\
\hline Filinia longiseta & $V=0,13 \mathrm{~b}^{3}$ & 3,29 & $V=0,13 \mathrm{~L}^{3}$ & 407529 & 116736 & 0.29 \\
\hline Eexarthre arra & $V=0,13 \mathrm{~d}^{3}$ & 0,23 & $v=0,13 \mathrm{~L}^{3}$ & 897197 & 201276 & 0.23 \\
\hline Brachionus angulatis & $V=0,12 \mathrm{~L}^{3}$ & 3.51 & $V=0,24 \mathrm{~b}^{3}$ & 309256 & 78870 & 0.26 \\
\hline B. anqularis CRBg & $V=0.128^{3}$ & 0,63 & $V=0,24 \mathrm{~b}^{3}$ & 502550 & 158103 & 0.32 \\
\hline B. calyciflorus & $V=0.12 \mathrm{~L}^{3}$ & 3.54 & $V=0.20 \mathrm{c}^{3}$ & 2996487 & 394323 & 0,33 \\
\hline B. Calyciflorus $A 89$ & $V=0,12 \mathrm{~L}^{3}$ & 0,53 & $V=0,20 \mathrm{i}^{3}$ & 1825888 & 5882230 & 0,32 \\
\hline B. calycifiorus $\mathrm{Bb}$ & $V=0,12 \mathrm{~L}^{\exists}$ & 0.88 & $V=0,20 \mathrm{~L}^{3}$ & 1321573 & $7097 B B$ & 0,53 \\
\hline B. calyciflorue UC & $V=0,12 \mathrm{~L}^{3}$ & 0,58 & $\nabla=0.20 \mathrm{~L}^{3}$ & 2175840 & 765084 & 0,35 \\
\hline B. calrciflorus $M B$ & $v=0,12 \mathrm{t}^{3}$ & 0,63 & $V=0,20 \mathrm{~L}^{3}$ & 2381415 & $911 \pm 64$ & 0,38 \\
\hline B. plicatilis COB & $V=0,12 \mathrm{~L}^{3}$ & 0,49 & $V=0,24 \mathrm{~L}^{3}$ & 1148055 & 281887 & 0.25 \\
\hline B. plicatlis is GS74 & $V=0,12 \mathrm{~L}^{3}$ & 8,39 & $V=0,24 \mathrm{~L}^{3}$ & 3818298 & 746543 & 0.20 \\
\hline Epiphanes brachionus & & & $V=0,34 \mathrm{~L}^{3}$ & 8590133 & 665361 & 0.078 \\
\hline Asplanchna priodanta & $V=0,23 \mathrm{~L}^{3}$ & 0,020 & $v=0,17 i^{3}$ & 11045417 & 308264 & 0,028 \\
\hline L. girodi & $V=0.23 \mathrm{~L}^{3}$ & 1.008 & $\nabla=0,15 \mathrm{~L}^{3}$ & 73444980 & 834337 & 0,012 \\
\hline ㅅ. briahtwelli & $V=0,23 \mathrm{~L}^{3}$ & 3,005 & $V=0,18 \mathrm{~L}^{3}$ & 103043048 & 644311 & 0,007 \\
\hline 4. herricki & $\nabla=0.23 \mathrm{~L}^{3}$ & 0,304 & $V=0,17 \mathrm{~L}^{3}$ & 155065768 & $734 \$ 96$ & 0,005 \\
\hline
\end{tabular}




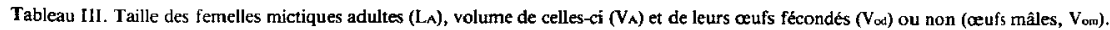
Table III. Size ( $\left.L_{i}\right)$ and volume $\left(V_{n}\right)$ of mictic females and volumes of their fertilized ( $\left.V_{o d}\right)$ or unfertilized $\left(V_{o m}\right)$ eggs.

a oeufs de durée (fécondés)

\begin{tabular}{|c|c|c|c|c|}
\hline Espèce & LA \pm e.s. $\mu \pi$ & VA $10^{6} \mathrm{wn}^{3}$ & Voa $10^{6} \mu^{3}$ & $V_{o d} / V_{n}$ \\
\hline Brachionus & & & & \\
\hline anqular is CR79 & $133,4 \pm 3,6$ & 0.570 & 0.202 & 0,36 \\
\hline B. calyciflorus $\mathrm{A} 89$ & $210,0 \pm 6,7$ & 1,857 & 0.598 & 0.33 \\
\hline Epiphanes brachionus & $305,5 \pm 12,8$ & 9.740 & 1,553 & 0,16 \\
\hline Asplanchna priodonta & $424,0 \pm 28,8$ & 13,124 & 1,345 & 0,10 \\
\hline A. girodi & $782,0 \pm 36,1$ & 72,166 & 4,086 & 0.057 \\
\hline A.brightwel1i & $837,0 \pm 46,9$ & 106,494 & 2,511 & 0,024 \\
\hline A. herricki & $962,0 \pm 72,5$ & 153,652 & 2,622 & 0.017 \\
\hline
\end{tabular}

b oeufs parthénogénétiques males

\begin{tabular}{|c|c|c|c|c|}
\hline Espèce & $\mathrm{LA} \pm$ e.s. $\mu \mathrm{m}$ & $V_{A} 10^{\epsilon} \mu m^{-3}$ & Vom $10^{\circ} 1 \mathrm{~m}^{3}$ & $V_{O m} / V_{A}$ \\
\hline \multicolumn{5}{|l|}{ Brachionus } \\
\hline anqularis CR79 & $131,5 \pm 2,8$ & 0,547 & 0,077 & 0.14 \\
\hline B. calyciflorus A89 & $205,9 \pm 9,9$ & 1.758 & 0,143 & 0.08 \\
\hline Epiphanes brachionus & $289,8 \pm 10,0$ & 8,299 & 0.321 & 0.04 \\
\hline
\end{tabular}

\section{LnVo}

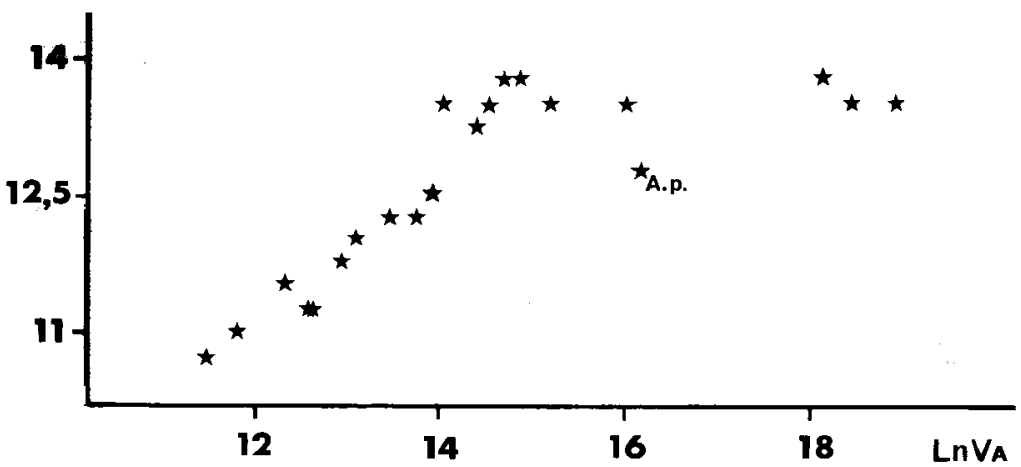

Fig. 2. Variations du volume de l'ceuf amictique $\left(V_{0}\right)$ en fonction du volume de la femelle adulte (VA) chez 21 populations de Rotifères planctoniques (valeurs moyennes exprimées en logarithmes népériens, Ln).

Fig. 2. Variations of the amictic egg volume $\left(V_{D}\right)$ in relation to the adult female volume $\left(V_{A}\right)$ in 21 planctonic rotifer populations (mean values in napierian logarithm). 
On obtient une mejlleure corrélation $\left(r^{:}=0,883\right.$, p $<0,01$ ) en limitant l'analyse aux seules espèces filtreuses herbivores tt la droite de régression est exprimée par l'équation: $V_{O}=3,62 V_{A} 0,816$.

Les mêmes calculs statistiques sur les seules populations récoltées in situ (10 espèces) fournissent des valeurs très proches: $V_{0}=1,42 V_{A} 0,884\left(r^{2}=\right.$ $0,946, p<0,01)$. Les données concernant les clones de laboratoire s'intègrent donc parfaitement aux données obtenues à partir des récoltes.

Un calcul plus affiné effectué sur les 280 valeurs (et non les seules valeurs moyennes comme précédemment) concernant les quatorze populations d'herbivores ( 12 espèces et 20 valeurs par population) conduit à des résultats similaires : $\mathrm{V}_{0}=3,27$ $\mathrm{V}_{\mathrm{A}} 0,811\left(\mathrm{r}^{2}=0,912, \mathrm{p}<0,001\right)$ (fig. 3).

2. Rapport du volume de l'ouf amictique au volume de la femelle adulte $V_{\mathrm{n}} / \mathrm{V}_{\mathrm{A}}$

Le rapport du volume de l' ceuf à celui de la femelle ovigère est élevé chez les petites espèces, telle que Keratella cochlearis où il atteint 0,46 . Il diminue considérablement chez les grandes Epiphanes et Asplanchna (Tableau II). Pour les autres espèces, en particulier celles appartenant au genre Brachionus, il varie de 0,20 à 0,53 . (it rapport étant considére comme stable pour une espiçe donnée, nous avons cherché à vérifier cette ass $\rightarrow$ tion chez Brachionus calyciflorus pour lequel nous connaissons la longueur moyenne de la carapace des femelles ovigères et celle du grand diamètre des ceufs dans un échantillon constitué de 42 couples de valeurs extraits de travaux de Hallbach (1970), Pourriot (1973) et du présent travail. Le volume des œufs a été estimé d'après la formule $V_{o}=0,30 \mathrm{~L}_{1}^{3}$, correspondant à une longueur relative du petit diamètre de l'ovoïde $\mathrm{L}_{2}=0,76 \mathrm{~L}_{1}$. Cette valeur moyenne a été obtenue à partir de mesures faites sur trois populations différentes.

En fait, l'amplitude de la variation du ratio $\mathrm{V}_{\mathrm{o}} / \mathrm{V}_{\mathrm{A}}$ chez $B$. calyciflorus est très large puisqu'il varie de 0,59 à 0,14 . Cette dernière valeur, anormalement basse, a été obtenue avec un clone cultivé à $10^{\circ} \mathrm{C}$ par Hallbach (1970). La distribution de fréquence de ces ratios est de type normal (fig. 4). Le volume de l'œuf est significativement corrélé à celui de la mère $\left(\mathrm{r}^{2}=0,816, \mathrm{p}<0,001\right)$ et la droite de régression est exprimée par l'équation : $\mathrm{V}_{0}=164$ $V_{A} 0.572$ (fig. 5). Si l'on exclut le cas aberrant cité ciavant, la pente de la droite est un peu supérieure à la précédente $(b=0,608)$.

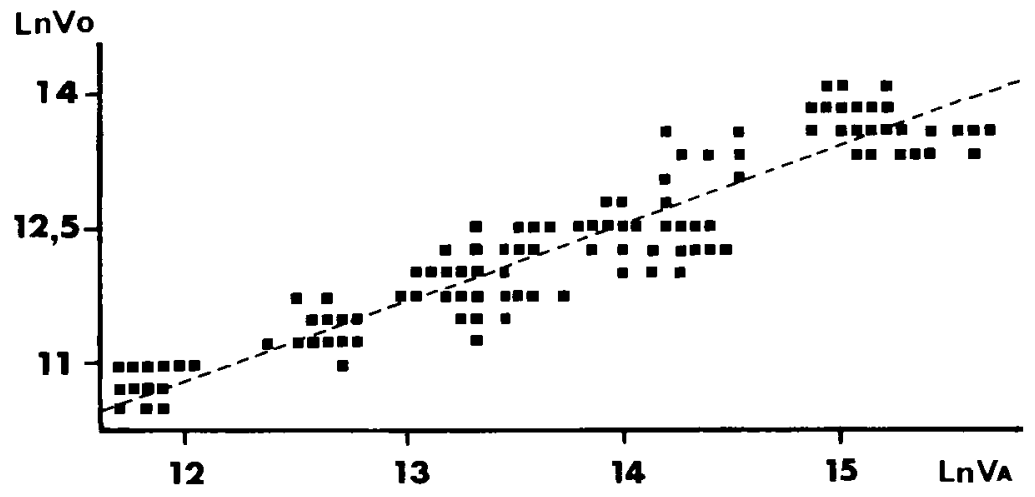

Fig. 3. Relation entre le volume de l'œu f amictique et celui de la femelle adulte chez 14 populations de Rotifères herbivores (logarithmes népériens de 280 valeurs).

Fig. 3. Relation between the egg volume and the adult female volume in 14 herbivorous rotifer populations (280 values in napierian logarithm). 


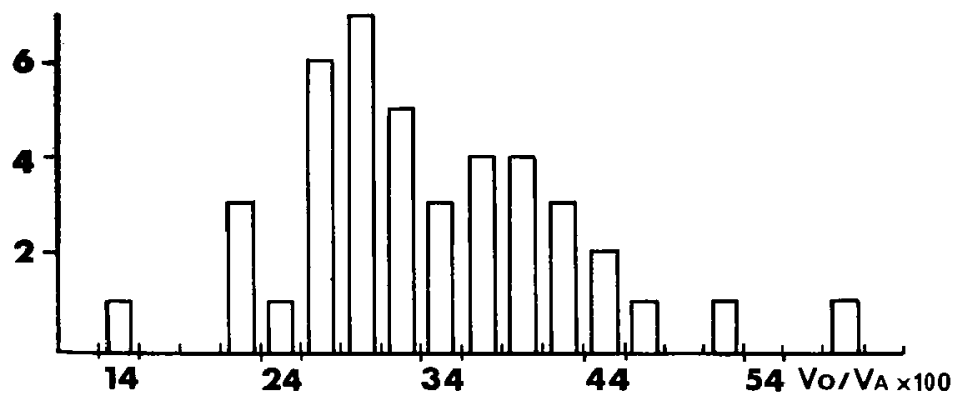

Fig. 4. Fréquence de répartition du rapport $\mathrm{V}_{\mathrm{o}} / \mathrm{V}_{\mathrm{A}}$ chez Brachionus calyciflorus.

Fig. 4. Distribution frequencies of the ratio $V_{0} / V_{A}$ in $B$. calyciflorus populations.

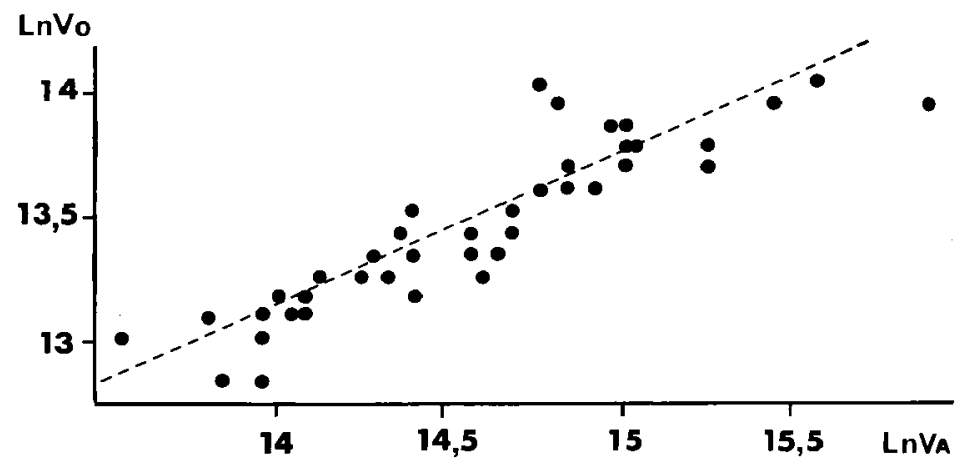

Fig. 5. Relation entre le volume de l'ouf amictique et celui de la femelle adulte chez Brachionus calyciflorus (valeurs moyennes exprimées en logarithmes népériens).

Fig. 5. Relation between the amictic egg volume and the adult female volume in Brachionus calyciflorus (mean values in napierian logarithms).

On notera enfin que la taille du nouveau-né n'est que de 15 à $20 \%$ supérieure à celle de l'œuf chez les espèces loriquées (par ex., à la naissance, la taille du jeune $B$. plicatilis $\mathrm{COB}$, est de $110,3 \mu \mathrm{m}$ pour une taille adulte de $168,1 \mu \mathrm{m}$ ) alors qu'elle est de $60 \%$ supérieure chez Epiphanes brachionus où elle atteint $207,2 \pm 7,2 \mu \mathrm{m}$ et représente environ $70 \%$ de la taille de l'adulte.

\section{Importance volumétrique des ceufs de durée et} des aufs mâles

Le volume de l'œuf de durée (Vod est significativement corrélé à celui de la femelle adulte $\left(\mathrm{r}^{2}=\right.$ $0,866 ; \mathrm{p}<0,001)$. La relation s'exprime par l'équation : $V_{o i}=705 V_{A}^{0,456}($ fig. 6). Comme précédemment, cette relation traduit le fait que le volume relatif de l'œuf (vs celui de l'adulte) décroît avec la 


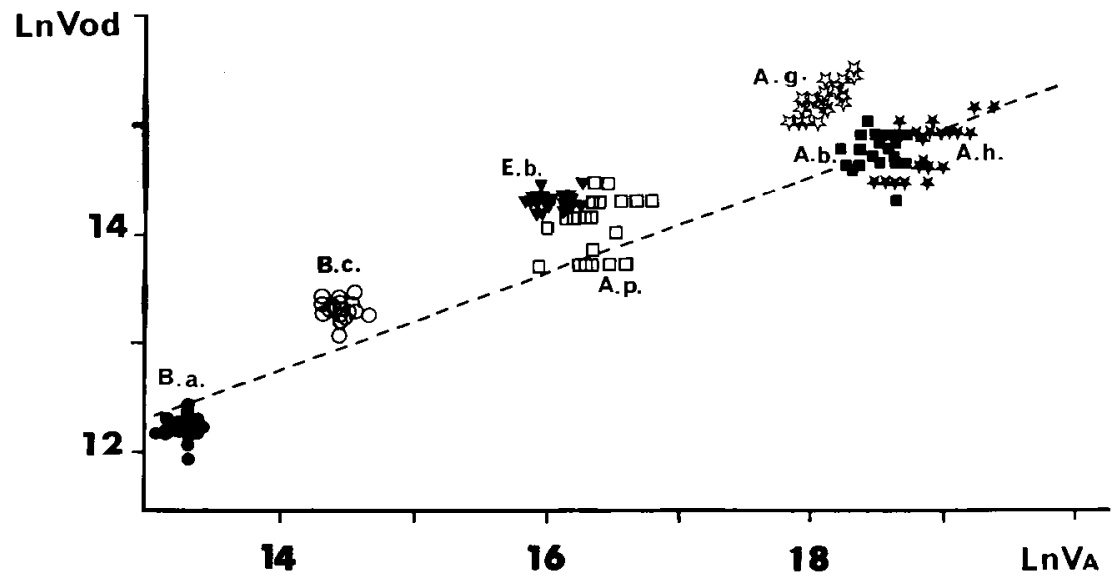

Fig. 6. Relation entre le volume de l'œuf de durée $\left(V_{\infty}\right)$ et celui de la femelle adulte $\left(V_{A}\right)$ chez sept espèces (les symboles correspondent aux initiales des noms de genre et d'espèce cités au tableau III). Les volumes sont exprimés en logarithmes naturels.

Fig. 6. Relation between the resting egg volume $\left(V_{o d}\right)$ and the adult female volume $\left(V_{A}\right)$ in seven species (symbols $=$ initial letters of the genus and species names; volumes in natural logarithms).

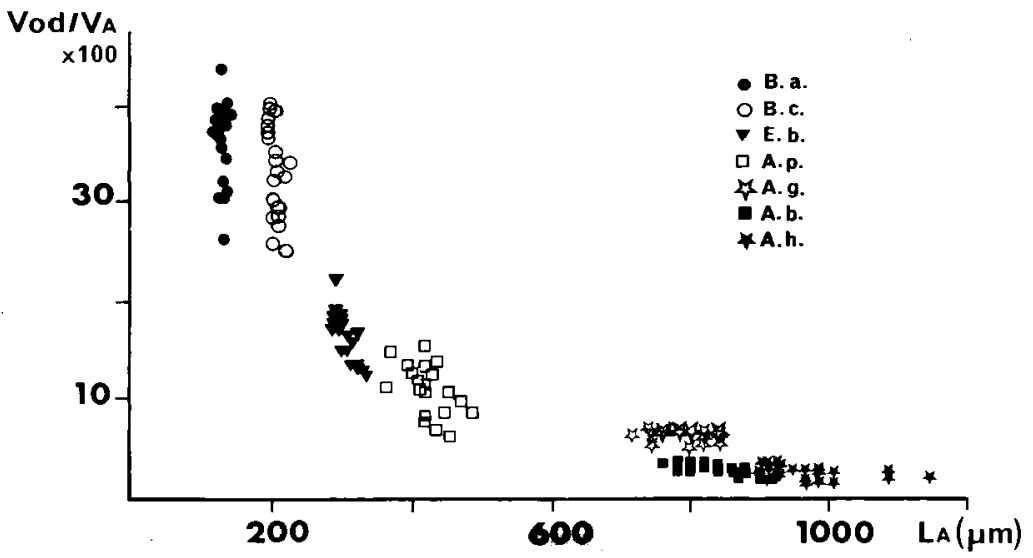

Fig. 7. Variation du rapport $\mathrm{V}_{\infty} / \mathrm{V}_{\mathrm{A}}$ en fonction de la longueur de la femelle adulte (LA) chez sept espèces de Rotifères.

Fig. 7. Variation of the ratio $V_{o d} / V_{A}$.in relation to the size of the adult female ( $L_{A}$ ) in seven rotifer species. 
taille de la femelle adulte et donc que les petites espèces ont les œufs les plus gros comparativement à leur propre volume (fig. 7). Toutefois, ce rapport des volumes respectifs semble se stabiliser chez les plus grandes espèces ( $A$. brightwelli et $A$. herricki) où le volume de l'œuf de durée ne s'accroît plus.

Si l'on fait abstraction de ces deux espèces, la corrélation est notablement améliorée $\left(r^{2}=0,947\right.$; $p$ $<0,001)$ et l'équation précédente devient : $V_{o d}=$ $92,3 \mathrm{~V}_{\mathrm{A}} 0,594$.

Une relation de même type est obtenue pour les œufs mâles des trois espèces algivores ayant produit suffisamment de femelles mictiques pour cette étude (Tableau III),

$$
\mathrm{V}_{\mathrm{om}}=77,7 \mathrm{~V}_{\mathrm{A}} 0,522 \text { (fig. 8). }
$$

Toutefois, le volume relatif $\left(V_{o m} / V_{A}\right)$ de ces petits œufs donnant naissance à des mâles nains est évidemment bien inférieur à celui des œufs femelles (Tableau IIIb).

\section{Discussion}

Les mesures de longueur, largeur et épaisseur de nos spécimens ( $a_{1}, a_{2}$ et a3 de Ruttner-Kolisko, 1977) nous ont conduit à modifier les formules simplifiées proposées par cet auteur ; généralement, nos mesures nous conduisent à une estimation supérieure du volume des adultes (Tableau II).
En outre, afin de faciliter la comparaison entre les différentes formes de Keratella cochlearis, nous avons utilisé une formule différente, basée sur la longueur de la carapace, en excluant l'épine postérieure qui est inexistante chez la forme tecta. Dans ce cas également, le volume calculé s'est avéré supérieur à celui obtenu en utilisant la formule de Ruttner-Kolisko.

Il s'ensuit que les rapports $V_{o} / V_{A}$ calculés selon nos critères sont parfois nettement inférieurs à ceux proposés par d'autres auteurs (cf. Pauli 1989). Iis nous semblent plus proches de la réalité. En effet, des rations $V_{0} / V_{A}$ supérieurs à 60 , voire $70 \%$ paraissent peu vraisemblables; ils supposent qu'avant la ponte, les organes de la femelle sont limités à un espace bien restreint, ce que l'observation ne confirme pas, même en supposant un léger gonflement par absorption d'eau lors de la sortic de l'œuf. Ainsi, les ratios très élevés fournis par Pauli (1977) pour Kellicottia longispina $(0,87$ et 0,75$)$ traduisent davantage une inadéquation de la formule utilisée pour le calcul du volume de la femelle (celui de l'œuf pose moins de difficultés) qu'une particularité de l'espèce !

Un autre point de vue semble devoir être remis en question : celui de la constance de ce ratio $\mathrm{V}_{\mathrm{o}} / \mathrm{V}_{\mathrm{A}}$ chez une espèce donnée. Chez Brachionus calyciflorus, ce rapport varie considérablement : l'amplitude de ces variations (fig. 4) est équivalente

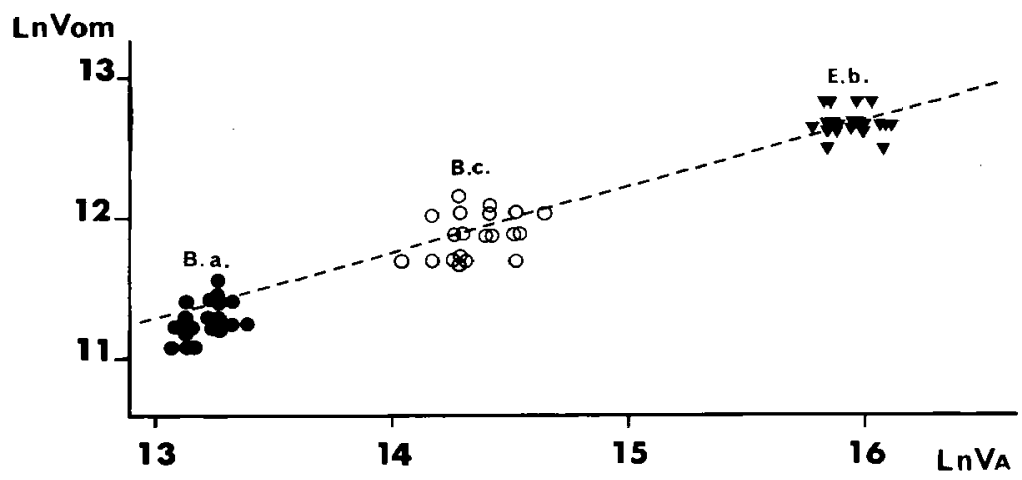

Fig. 8. Relation entre le volume de l'cuf mäle $\left(V_{t m}\right)$ et cẹlui de la femelle adulte $\left(V_{A}\right)$ che $c$ trois espèces planctoniques. Volumes exprimés en logarithmes naturels.

Fig. 8. Relation between the male egg volume $\left(V_{\mathrm{om}}\right)$ and the adult female volume $\left(V_{\mathrm{A}}\right)$ in three planktonic species (volumes in natural logarithms). 
à celle de l'ensemble des espèces de taille faible ou moyenne ( $<350 \mu \mathrm{m}$, Tableau II) même en excluant les valeurs extrêmes (et ce, quelle que soit la formule utilisée pour le calcul du volume de l'adulte). Etant donné la relation allométrique liant ces deux variables, $V_{0}$ et $V_{A}$, il paraît logique que ce rapport décroisse avec la taille de la femelle adulte et ne soit donc pas constant. Ces deux variables dépendent, de plus, non seulement de la température mais aussi de la nourriture (Sarma \& Ramakrishna 1987), ces deux facteurs pouvant avoir des effets conjugués ou opposés selon les conditions de milieu.

Les valeurs des exposants calculés pour les différentes relations établies dans le présent travail pour les espèces herbivores sont toutes proches de la puissance $3 / 4$ qui lie généralement la masse de l'œuf à celle de la mère (Peters 1983). Il est d'ailleurs difficile de concevoir qu'il puisse en être autrement chez des animaux où l'investissement reproductif est notablement supérieur à l'investissement de croissance corporelle. L'explication avancée par Pauli (loc. cit.) pour justifier la faible valeur de l'exposant qu'il obtient consiste à attribuer aux rotifères une faible teneur en biomasse sèche. La pertinence de cet argument paraît faible au vu des poids secs relatifs (pourcentages du poids frais) récapitulés par Pauli (loc. cit., Tableau II). Ceux-ci varient de 3,2 à $43,2 \%$ chez les espèces herbivores avec des valeurs situées plus fréquemment autour de 9 a $20 \%$ et qui ne s'écartent guère de la norme.

Il en va tout autrement pour les Asplanchna, espèces de grande taille, où la masse des organes internes n'a pas crû dans les mêmes proportions que le tégument d'où leur apparence de sac peu rempli et un poids sec n'atteignant en moyenne que $4 \% \mathrm{du}$ poids frais (Dumont et al. 1975, Pauli 1989). Il est à noter en outre que, contrairement aux autres espèces citées ci-avant, les Asplanchna sont dépourvues d'intestin et ovovivipares, l'embryon se développant dans l'oviducte de la mère. Il n'est donc pas étonnant que la taille de l'œuf soit limitée et ne suive plus la relation allométrique établie précédemment pour les espèces herbivores.

Le relativement faible ratio observé chez les $A$. priodonta fixées au formol (fig. 2) pourrait résulter d'une légère contraction du corps contuisant à sousestimer son volume par rapport à celui des autres espèces mesurées à l'état vivant.
Il ressort de l'ensemble de ces observations que les trois types d'œufs pondus par les rotifères suivent la même loi générale, les plus petites espèces ayant les cufs les plus volumineux par rapport à leur propre taille. Cependant, la valeur de la pente de l'équation exprimant cette loi est quelque peu inférieure pour les œufs de durée et les œufs mâles. Il est probable que ceci reflète l'existence de contraintes sélectives différentes dans les trois cas. Après la ponte, les œufs de durée sédimentent et subissent généralement une période de diapause avant d'éclore dans des conditions précises souvent propices à une croissance rapide (Pourriot \& Snell 1983). Par ailleurs, la croissance est quasiment nulle chez les mâles nains, à durée de vie courte et nage rapide.

\section{Remerciements}

Les auteurs remercient P. Clément et E. Wurdak, le premier pour la photographie au MEB de Brachionus sericus et tous les deux pour l'obtention de la souche d'Asplanchna herricki, récoltéc dans le Vermont (USA).

Travaux cités

Dumont (H. J.), Van de Velde (I.) \& Dumont (S.). 1975. - The dry weight estimate of biomass in a selection of Cladocera. Copepoda, and Rotifera from the plankton, periphyton, and benthos of continental waters. Oecologia $19: 75-97$.

Hallbach (U.). 1970. - Die Ursachen der Temporalvariation von Brachiomus calyciflorus Pallas (Rotatoria). Oecologia 4 : 262-318.

Pauli (H.-R.). 1989. - A new method to estimate individual dry weights of rotifers. Hydrobiologia 186/187 : 355-361.

Peters (R.H.). 1983. - The ecological implications of body size, Cambridge University Press : $329 \mathrm{p}$

Pourriot (R.). 1973. - Rapports entre la temperature, la taille des adultes, la longueur des cufs et le taux de développement embryonnaire chez Brachionus calyciflorus Pallas (Rotifère). Annis. Hydrobiol. 4 : 103-115.

Pourriot (R.) \& Snell (T.W.). 1983. - Resting eggs in rotifers. Hydrobiologia 104 : 213-224.

Ruttner-Kolisko (A.). 1977. - Suggestions for biomass calculation of plankton rotifers. Arch. Hydrobiol, Beih., Ergbn. Limnol. 8 : 71.76.

Sama (S.S.S.) \& Ramakrishna Rao (T.). 1987. - Effect of food level of body size and egg size in a growing population of the rotifer Brachionus patulus Müller. Arch. Hydrobiol. 111 : 245-253. 